\title{
Notch Ligand Presenting Acellular 3D Microenvironments for ex vivo Human Hematopoietic Stem-Cell Culture made by Layer-By-Layer Assembly**
}

\author{
Jungwoo Lee and Nicholas A. Kotov*
}

Development of in vitro human hematopoietic stem cell (HSC) niches that can recapitulate the bone marrow and thymus function is imperative for understanding the fundamental biology of human hematopoiesis. Currently most information of HSC self-renewal and lineage commitment has been primarily derived from murine studies where a substantial knowledge gap in humans still exists. Such established functional tissue analogues can also greatly serve as a valuable tool to identify specific signaling factors and their precise roles in hematopoiesis by systematically manipulating major experimental parameters.

The thymus is a specialized organ providing unique environments for the development of T-lymphocytes from bone-marrow-derived HSCs. ${ }^{[1]}$ Although the precise nature of the thymic environment inducing T-cell differentiation remains uncertain, it has been identified that stromal cells and 3D microarchitecture play a critical role from numerous studies of ex vivo HSC cultures. ${ }^{[2-4]}$ For example, thymic stromal cells activate various cell-signaling processes through secreting growth factors and expressing membrane-bound ligands. Their 3D organization creates a distinctive intrathymic 3D microarchitecture that coordinates the aforementioned signaling milieus. Thymocytes passing through the interstices of the 3D network of thymic stroma undergo extensive physical contacts with stromal cells, which is the main mechanism to promote $\mathrm{T}$-cell differentiation and maturation. ${ }^{[5,6]}$ Due to such complexity, until recently the only reliable in vitro culture model for successful T-cell differentiation was the fetal thymus organ culture (FTOC), which is composed of a $3 \mathrm{D}$ reaggregated dissection of fetal thymic tissues. ${ }^{[5-7]}$

[*] Prof. N. A. Kotov, J. Lee

Departments of Biomedical Engineering, Chemical

Engineering, and Material Science \& Engineering

University of Michigan

3074 H. H. Dow Building

2300 Hayward Street

Ann Arbor, MI 48109 (USA)

E-mail: kotov@umich.edu

[**] Authors thank Prof. Irwin Bernstein for his valuable comments on the manuscript and acknowledge the staff of the Electron Microscopy Analysis Laboratory.

몽 Supporting Information is available on the WWW under http:// www.small-journal.com or from the author.

DOI: $10.1002 / \mathrm{smll} .200801242$
Notch signaling is a major cell-cell signaling pathway determining HSC fate in the bone marrow and thymus. The signaling is activated when a notch receptor on HSCs directly contacts the delta and jagged families of notch ligands present on the stromal cell membrane (Figure 1A). The expression of ligands and receptors is tightly regulated to balance between self-renewal and differentiation of HSCs in the bone marrow, and to promote T-cell lineage commitment and maturation in the thymus. ${ }^{[8,9]}$ The importance of notch signaling in T-cell development was demonstrated with a $2 \mathrm{D}$ ex vivo co-culture model of HSCs and OP9-DL-1 stromal cells expressing the delta-like1 (DL-1) notch ligand. ${ }^{[10]}$ For example, the OP9DL-1 cells successfully supported T-cell differentiation of HSCs, whereas regular OP9 cells only induced B-cell differentiation. ${ }^{[11,12]}$ Although these in vitro culture models greatly contributed to the understanding of HSC biology, their purely biological nature presents inherent limitations including technically burdensome preparation, low yields, and highly variable outcomes.

Recent advances of biomaterial process engineering enabled the recapitulation of the hematopoietic process to some extent on biomaterial-based in vitro culture environments. ${ }^{[13]}$ Simple and directed preparation of artificial microenvironments was exceptionally beneficial to improve experimental controllability and reproducibility. It also allowed the systematic manipulation of experimental parameters, which in turn deepen the fundamental understanding of thymic signaling effects. For instance, 3D co-culture models of bone marrow and thymus established on 3D scaffolds significantly promoted B-cell ${ }^{[14]}$ and $\mathrm{T}$-cell ${ }^{[15,16]}$ differentiation of HSCs compared to the conventional monolayer cultures. In addition, these studies highlighted the importance of the proper design of a 3D scaffold to obtain the optimum range of pore size and density so as to maximize ex vivo HSC development. Nevertheless, 3D co-culture models retain several critical drawbacks: i) difficulty in control of the growth of stromal cells in 3D substrates; ii) requirement of an extra step to isolate a pure population of HSCs when the culture needs to be characterized; iii) potential phenotypic change of feeder cells due to enhanced cell-cell and cell-matrix interactions in 3D culture conditions. ${ }^{[17]}$

A different promising approach was stromal cell-free T-cell differentiation, which presents multiple advantages such as better control over cell interactions and signaling, and efficient isolation and characterization of HCSs. The most 
A

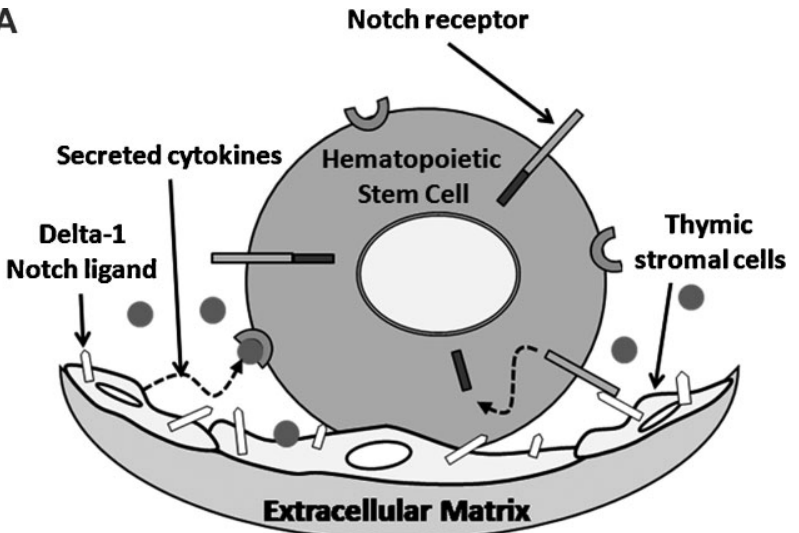

B

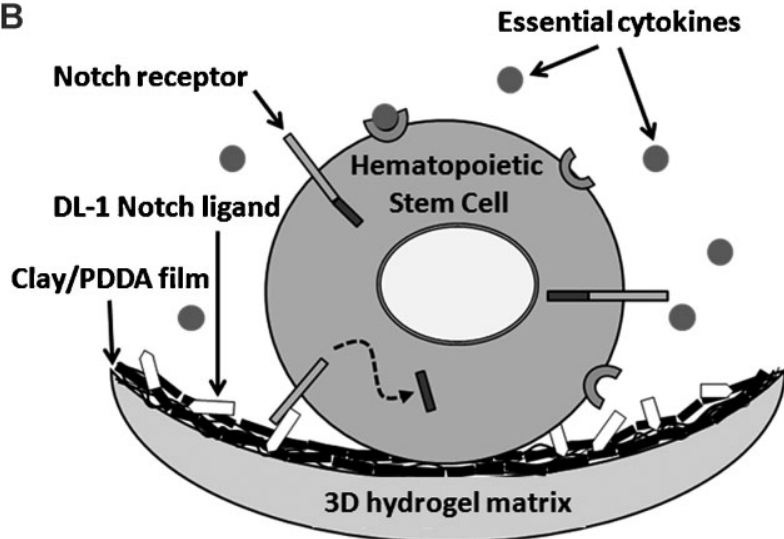

Figure 1. Schematic of a 3D thymic microenvironment and an artificial 3D microenvironment mimicking a real environment. A) In the thymus, 3D-organized thymic stromal cells present membrane-bound notch ligands and secrete soluble cytokines for the T-cell differentiation of HSCs. B) ICC topology provides a 3D intrathymic microstructure and the multicomponent LBL film (i.e., a monolayer of DL-1 notch ligands on top of 5 bilayers of clay/PDDA film) substitutes thymic stromal cell functions in addition to soluble cytokines.

successful system utilized engineered DL-1 notch ligands in addition to essential cytokines. For instance, individual DL-1 notch ligands were immobilized either by physical absorption on the bottom of a well-plate ${ }^{[18-20]}$ or by chemical conjugation to microcarriers, ${ }^{[21]}$ and successfully supported T-cell development. Moreover, the readily adjustable ligand density of this technique distinguished the different effect of notch signaling intensity to determine the fate of HSC differentiation. ${ }^{[19,22,23]}$ Even though notch ligands presenting acellular microenvironments are an attractive approach to create in vitro HSC niches in a convenient and consistent manner, it has remained only in 2D substrates, probably due to the absence of suitable 3D substrates and coating techniques.

In this report, we introduce our efforts to combine 3D microarchitecture and notch ligand effects for in vitro HSC cultures under the hypothesis that 3D inverted colloidal crystal (ICC) scaffolds coated with DL-1 notch ligand would considerably substitute for the structure and function of thymic stroma in addition to essential cytokines (Figure 1B). An appropriate design and dimension of $3 \mathrm{D}$ scaffolds that can induce intense and proper configurations of cell-cell contacts is an essential part of the in vitro recapitulation of functional thymic niches. A recently developed unique design of ICC topology was used as a framework to mimic the 3D thymic microarchitecture. ${ }^{[24,25]}$ A layer-by-layer (LBL) molecular assembly technique was utilized to immobilize DL-1 notch ligands on the surface of the ICC pores since the solutionbased LBL coating procedure can be readily applied to the surface coating of complex 3D porous substrates while minimizing distortion of protein structures. ${ }^{[26]} \mathrm{We}$ investigated the effectiveness of 3D ICC topology and the bioactivity of LBL-immobilized DL-1 notch ligands in ex vivo T-cell development of human HSCs.

The basic structure of an ICC scaffold consists of highly organized and uniformly-sized spherical pores that are wellinterconnected with adjacent pores. ${ }^{[2]}$ It facilitates oxygen and nutrient transport as well as homogeneous cell distribution within the scaffold. ${ }^{[27,28]}$ Under a dynamic culture condition, floating cells can be temporarily entrapped in an ICC pore before moving into neighboring pores due to the finite size and number of interconnecting channels, which promote extensive physical contacts between floating HSCs and the surface of the ICC scaffold where stromal cells grow or become coated with specific cell-signaling molecules. ${ }^{[25]}$ In this study, polystyrene microspheres with a diameter of $100 \mu \mathrm{m}$ were used to construct colloidal crystal templates (Figure 2A-C) considering the
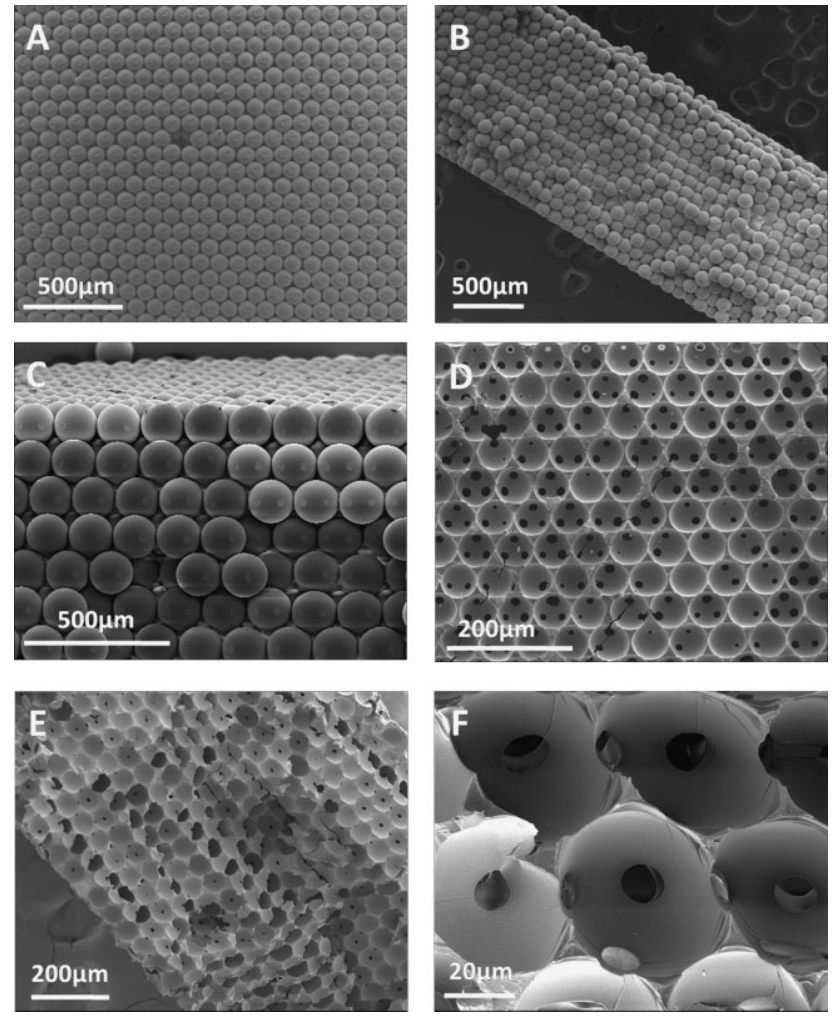

Figure 2. SEM images of colloidal crystals and hydrogel ICC scaffolds. A-C) Different sides of colloidal crystals prepared with polystyrene microspheres $100 \mu \mathrm{m}$ in diameter; A) bottom, B) cross section, and C) tilted cross section. D-F) Different sides of ICC scaffolds; D) bottom and E,F) different magnification of cross sections. Note that hydrogel ICC scaffolds were dehydrated before imaging, which caused shrinkage of pore size. 


\section{communications}

dimension of HSCs $(<10 \mu \mathrm{m})$. Final polyacrylamide hydrogel ICC scaffolds had $110-\mu \mathrm{m}$ - and approximately $25-30-\mu \mathrm{m}$ diameters of pore and interconnecting channel size, respectively (Figure 2D-F).

We utilized a LBL molecular assembly technique to make the 3D surface coating of the DL-1 notch ligand. The main driving forces for LBL coating are electrostatic, van der Waals, and hydrogen bonding interactions between oppositely charged polyelectrolytes dissolved in water. ${ }^{[26]}$ This solution-based coating technique can effectively modify the surface of the complex porous 3D ICC geometry since the fluid transport in and out of the scaffold is well maintained. In addition, the versatile nature of a LBL assembly technique enables a spatially arranged multicomponent surface coating including a large variety of proteins. ${ }^{[29]}$

Previously we demonstrated the LBL coating of hydrogel ICC scaffolds with a multilayer of clay and poly(diallyl dimethylammonium chloride) (PDDA) that promoted association of bone marrow stromal cells on the scaffolds. ${ }^{[14,25]}$ The same clay/PDDA film was used as a bottom layer to provide a stiff/rigid layer on the hydrogel matrix, improving HSC contacts. The pre-deposited LBL film also provides a better environment for the following DL-1 notch ligand coating by forming higher electrostatic charges than the bare hydrogel surface. As a result the monolayer of DL-1 notch ligands was prepared on top of five bilayers of clay/PDDA film that formed on the surface of the hydrogel ICC scaffold. To characterize the quality of the 3D LBL coating, the fragment crystallizable $(\mathrm{Fc})$ portion of the ligand was tagged with a secondary antibody conjugated to a fluorescent dye before coating and visualizing under a confocal microscope. As shown in Figure 3, DL-1 notch ligands were coated well on the surface of the ICC scaffold but a slightly weaker fluorescent intensity was observed in the side pores, which would be caused by the relatively less diffusive environment of the interior pores.

The alternative layer of the organic and inorganic composite is mechanically compatible with polyacrylamide hydrogel, well-known for its non-fouling property, and

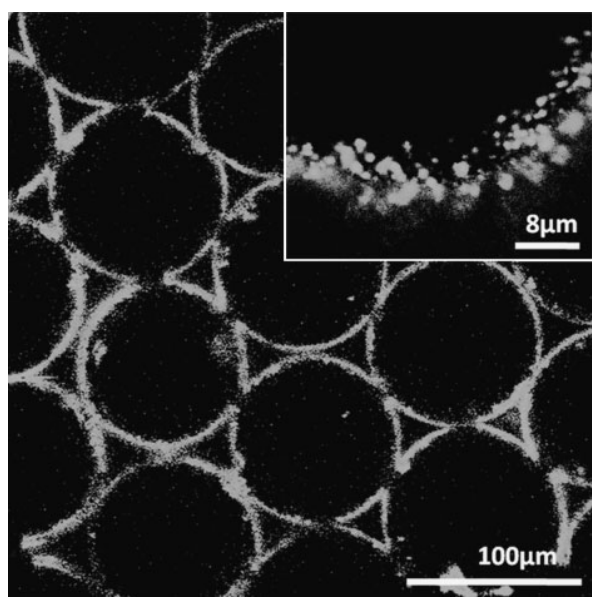

Figure 3. Confocal image of a DL-1 notch-ligand-coated hydrogel ICC scaffold. For imaging purposes, DL-1 notch ligands were bound to antibodies conjugated to FITC. supports steadfast immobilization of DL-1 notch ligands. It is an important feature for receptor-ligand-based cell signaling because ligands existing in a soluble state, although they can bind to receptors and activate signal transduction, normally undergo rapid internalization into a cell, which in turn considerably reduces the intensity and duration of cell signaling. ${ }^{[30]}$ Other investigators demonstrated that the surface-tethered epidermal growth factor (EGF) more effectively promoted cell spreading and survival than a saturating concentration of soluble EGF due to the longer duration of intense ligand-receptor interactions. ${ }^{[31]}$ Similarly it has been shown that DL-1 notch ligands are required to be immobilized to activate the proper level of notch signaling. ${ }^{[19,32]}$

The LBL adsorption technique also affords the scaffolds to retain high stability and activity of the notch ligands since the LBL coating is conducted in a mild condition while maintaining pristine protein structures. ${ }^{[33-35]}$ Moreover LBL-immobilized ligands can have a moderate level of flexibility because, although individual clay platelets provide local mechanical strength, the overall LBL film provides softness and elasticity found in the extracellular matrix (ECM) due to the hydrated polymeric component. It was shown that mechanical properties of a clay/PDDA film are strongly decreased in a humid environment. ${ }^{[36]}$ The flexibility of immobilized ligands is essential for the proper development of the ligand docking with the receptor for intracellular signal activation. ${ }^{[37]}$ Hence, the behavior of LBL-coated DL-1 ligands would be quite different when compared to ligands directly adsorbed on a solid surface. Considering that cellular ligands are actually embedded in a flexible plasma membrane, it is a more realistic and effective approach. Another advantage of LBL adsorption is its time-effectiveness. Normally it takes several days to reach the confluence of a stromal cell layer on 3D scaffolds, however, a LBL coating can create a similar environment in a few hours. All these features differentiate the LBL coating of notch ligands from other techniques.

As a first step, we tested the 3D ICC topological effect by culturing bone-marrow- or umbilical-cord-blood-derived mononuclear cells in a rotary cell culture vessel. Dynamic culture condition was utilized not only to create a more realistic physiological environment but also to maximize the role of specifically designed ICC geometry. Continuous convective media flow through ICC pores is the main driving force for cellular motility within the scaffold, which is similar to an in vivo environment. The cross-sectional image of an ICC scaffold after 15 days of culture shows that a significant number of cells reside in the inner pores of the scaffold (Figure 4A). This demonstrates that the mononuclear cells were deeply transported into the ICC scaffold and that the LBL surface modification promoted cell adhesion. Since HSCs are only a small fraction of mononuclear cells (1-2\%), most cells were non-HSCs. However, interestingly we could observe the development of pre-erythrocytes attaching on the pore surface, which might be the result of activated notch signaling. ${ }^{[20]}$ In addition, we observed dendritic-like cells scavenging across ICC pores, which vividly captured an in vivo 3D bone marrow microenvironment (Figure 4B).

As a next step we isolated CD34+/CD4- HSCs from the mononuclear cells and cultured then under the same 


\section{small}
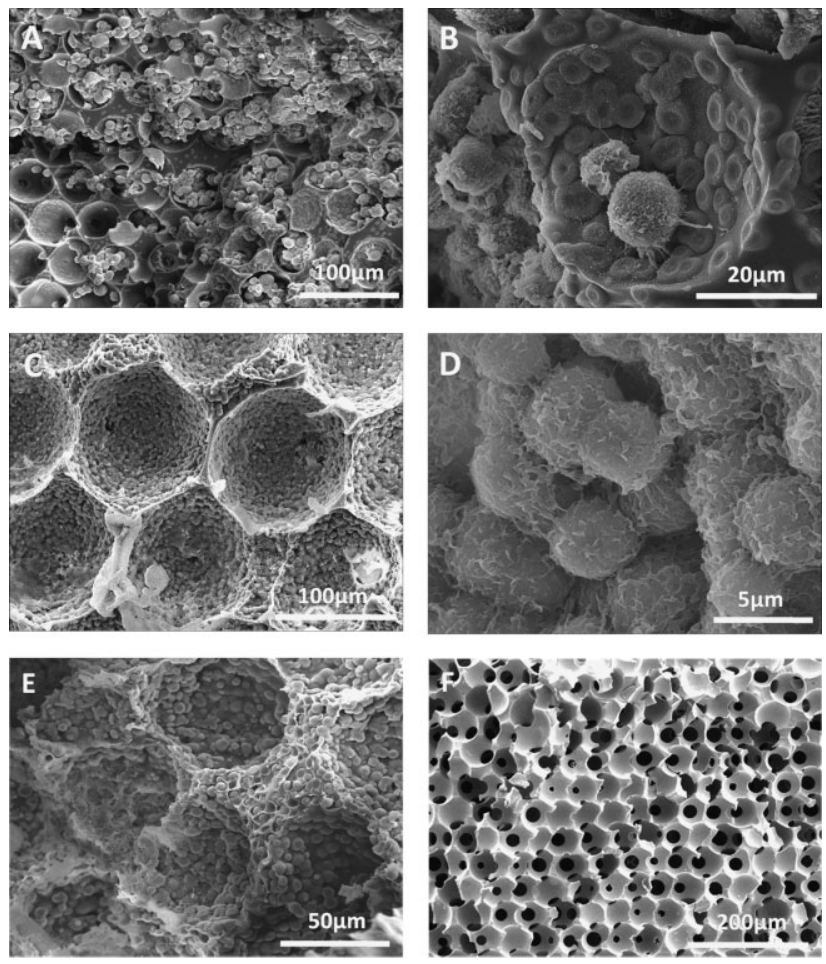

Figure 4. SEM images after 15 days of culture. A,B) Mononuclear cells. A) Various cell types coexisted deep inside ICC pores. B) Development of pre-erythrocyte associating with the LBL-coated surface and migrating dendritic-like cells across pores. It well-recapitulated the bone marrow microenvironment. C,D) Isolated CD34+ cells from bonemarrow-derived mononuclear cells. C) The DL-1 notch-ligandimmobilized pore surface was densely covered with cells. Restricted cellular population mimicked the thymic microenvironment. D) Due to similar morphology and size, it is hard to distinguish developing Tlymphocytes from CD34+ HSCs under SEM. E,F) Control experiments of 15-day-cultured CD34+ cells. E) ICC scaffolds could support cell adhesion without a DL-1 notch ligand coating. F) Without a clay/PDDA LBL coating (bare hydrogel), ICC scaffolds could not allow any cell adhesion.

conditions. Utilizing CD34+ HSCs is important to mimic the thymic niches because an initial stage of the cellular population entering into the thymus is mostly bone-marrowderived HSCs. In addition, it is necessary to deprive CD4+ cells from the initial cell population to confirm the bioactivity of LBL-coated DL-1 notch ligands since notch-1 signaling induces CD4+ T-cell lineage commitment at the late stage of differentiation. ${ }^{[38,39]}$ Since a magnetic-activated cell sorting (MACS) method usually cannot separate other cell types with $100 \%$ efficiency, we first depleted CD4+ cells from mononuclear cells and then enriched CD34+ cells (see Supporting Information). Small diameter HSCs $(<10 \mu \mathrm{m})$ readily moved deep into ICC scaffolds and intimately associated with the surface of DL-1 notch-ligand-coated ICC pores. After 14 days of culture, the pore surface was densely covered with cells (Figure 4C). This result inferred that HSCs proliferated multiple times subsequent to entering into the ICC scaffolds. Notch signaling would be activated on the surface-associated HSCs, which in turn stimulated T-cell lineage commitment and differentiation. However, it was hard to distinguish differentiated T-lymphocytes from HSCs under scanning electron microscopy (SEM) due to their similarity in morphology, size, and shape (Figure 4D). In the absence of a DL-1 notch ligand coating, mononuclear cells readily adhered to the surface of ICC scaffolds, which was comparable to the notch-ligand-coated scaffolds (Figure 4E). However, without a clay/PDDA LBL surface coating, hydrogel ICC scaffolds could not support cell adhesion and most pores appeared empty (Figure 4F). Although a few cells were entrapped in the pores during dynamic cell culture, most of them were released during the sample preparation procedure due to the interconnected open porous ICC geometry.

In order to confirm T-cell differentiation of HSCs, we characterized the progression of differential stage-specific surface marker expression for 4 weeks under confocal microscopy. The transparent polyacrylamide hydrogel matrix was exceptionally beneficial to imaging cells residing deep inside of 3D ICC scaffolds up to $500 \mu \mathrm{m}$ in depth (Figure 5A and Supporting Information, Movie). Besides the CD34 molecule, CD117 (so-called a C-kit receptor) is another representative surface marker of primitive HSCs that also maintains the earliest thymocyte progenitors. Examination of ICC cultures on days 15 and 22 showed the continued presence of CD117, which proved that our ex vivo 3D culture system successfully supported self-renewal or maintaining an undifferentiated population of HSCs. In addition, we could observe the presence of T-cell receptor (TCR) $\alpha$ and CD3 molecules (Figure 5B and C). TCR is a complex of integral membrane proteins consisting of $\alpha / \beta$-heterodimers associated with CD3 molecules. Pre-TCR expression is one of initial signs in T-cell lineage commitment and development in the Thymus. ${ }^{[6]}$
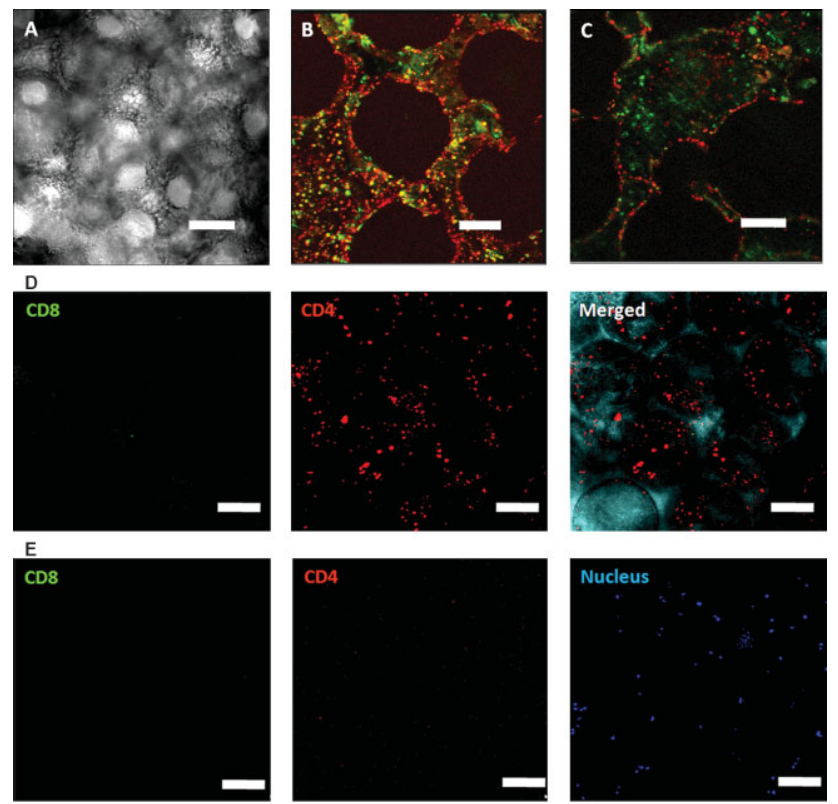

Figure 5. A) Typical transmission image of a CD34+ HSC growing ICC scaffold after 15 days of culture. Representative confocal images of B) CD117 (green) and CD3 (red) after 15 days of culture, and C) CD117 (green) and TCR $\alpha$ (red) after 22 days of culture. (Scale bar $=40 \mu \mathrm{m}$.) $D, E) C D 4$ and CD8 surface marker analysis after 28 days of culture. D) DL-1 notch-ligand-coated ICC scaffolds induced CD8-/CD4+ T-cell development. E) Neither CD4 nor CD8 was detected on the ICC scaffold in the absence of the DL-1 notch ligand coating. (Scale bar $=80 \mu \mathrm{m}$.) 


\section{communications}

Therefore, these data substantiate the preserved bioactivity of LBL-coated notch ligands so as to promote pre-T-cell differentiation of HSCs.

We further investigated the expression of CD4 and CD8 molecules over the longer period of ICC cultures to identify double positive and single positive differentiation stages. On 28 days of culture we could observe some CD4+ but CD8cells (Figure 5D). However, they would not be functional CD4 helper T-cells because in our system the major histocompatibility complex (MHC) molecules-important factors involved in cell screening processes, that is, positive and negative selection - were missing. Generally more than $95 \%$ of thymocytes undergo apoptosis during the screening processes, ${ }^{[1]}$ but we did not observe severe cell apoptosis during the culture. Without a DL-1 notch ligand coating, we could not observe either CD4 or CD8 molecules at 28 days of culture (Figure 5E). However, the culture continuously expressed a CD34 surface marker for 14 days, which infers that $3 \mathrm{D}$ dynamic cell culture stimulates ex vivo HSC expansion without notch signaling. Another important aspect was that ECM-like molecules progressively developed on the surface of cell/scaffold over the culture period, which reduced the quality of surface marker analysis. For that reason, 28-day-cultured samples showed relatively weaker fluorescent signals than the samples cultured shorter periods of time.

Although our 3D culture model demonstrated promising features to create in vitro HSC niches, it was difficult to rapidly isolate a large enough number of cells from ICC culture due to strong cellular association with the LBL-coated surface and the complex ICC geometry limiting the release of cells. Flow cytometric analysis of individual cells is critical to quantitatively corroborate the study of cell differentiation pathways accompanied with qualitative confocal surface marker characterization. To overcome these drawbacks, the development of effective cell harvesting methods needs to be further investigated.

Potentially the LBL coating system can also be applied to recapitulate the other function of stromal cells: releasing soluble growth factors. Since multiple functional components can be sequentially incorporated into a growing film, a compartmentalized LBL film consisting of soluble signalingmolecule-embedded bottom layers and ligands presenting top layers can be prepared on the surface of 3D scaffolds. Diffusion of soluble factors can be regulated by hydrolytically degradable polymer components. ${ }^{[40]}$ In addition, a recently discovered exponential LBL system can be used for loading and unloading soluble signaling molecules. ${ }^{[41,42]}$ As a result, such multicomponent and functional LBL films on 3D scaffolds can deliver both insoluble and soluble signaling molecules to the cells, which in turn significantly improves the creation of artificial 3D HSC niches in a simple and effective way.

In conclusion, we successfully extended DL-1 notch ligand coating onto 3D ICC scaffolds utilizing a LBL surface modification method and verified its biological significance as the T-cell differentiation of HSCs. The LBL technique showed distinguished advantages in creating an artificial 3D thymic stromal cell layer such as convenience, versatility, high controllability with nanoscale resolution, and capacity to build a multifunctional complex structure. These characteristics can also be applied to other immunologically relevant complex systems as a valuable tool to investigate human HSC biology from the molecular to the tissue level. Therefore, 3D LBL scaffolds can significantly contribute to the realization of ex vivo production and clinical usage of immunologically important cells.

\section{Experimental Section}

Hydrogel ICC scaffold preparation: Hydrogel ICC scaffolds were prepared following the previously reported method. ${ }^{[25]}$ Briefly, colloidal crystal (CC) templates (diameter $=6.5 \mathrm{~mm}$ and height $=0.5-1 \mathrm{~mm}$ ) were constructed with polystyrene microspheres with a diameter of $100 \mu \mathrm{m}$ (Duke Scientific). After thermal annealing at $120^{\circ} \mathrm{C}$ for $4 \mathrm{~h}$, a hydrogel precursor solution composed of $30 \%(\mathrm{w} / \mathrm{w})$ acrylamide monomer, $5 \%(\mathrm{w} / \mathrm{w})$ bisacrylamide crosslinker, and $0.1 \%(\mathrm{v} / \mathrm{v}) \mathrm{N}, \mathrm{N}, \mathrm{N}, \mathrm{N}$-tetramethylethylenediamine was infiltrated into the $C C$ by centrifugation at $5800 \mathrm{rpm}$ for $10 \mathrm{~min}$, and then polymerized by adding $1 \%(\mathrm{w} / \mathrm{w})$ potassium peroxide initiator solution. The CC-containing hydrogel was cut out and immersed in tetrahydrofuran for $24 \mathrm{~h}$ to remove the template. Finally the hydrogel ICC scaffolds were thoroughly washed with deionized water.

LBL surface modification: The surface of hydrogel ICC scaffolds was coated with five bilayers of a negatively charged $0.5 \%(\mathrm{w} / \mathrm{w})$ clay nanoparticle dispersion and positively charged $0.5 \%(\mathrm{w} / \mathrm{w})$ PDDA solution (Sigma, $M W=200000$ ) utilizing a LBL method. Scaffolds were alternatively immersed in each component solution for $15 \mathrm{~min}$ and rinsed with deionized water for $30 \mathrm{~min}$. Starting and finishing layers were PDDA. Engineered DL-1 notch ligands consisting of the extracellular domain of the delta 1 ligand fused to the $\mathrm{Fc}$ domain of human immunoglobin $\mathrm{G}^{[20]}$ were gifted from Prof. Irwin Bernstein's lab (University of Washington). A monolayer of the DL-1 notch ligand coating was prepared on top of the clay/ PDDA film by immersing the scaffold in a $10 \mu \mathrm{g} \mathrm{mL}^{-1}$ of DL-1 notch ligand solution for $30 \mathrm{~min}$. For imaging purposes, the Fc portion of DL-1 notch ligands was bound with secondary antibodies conjugated to fluorescein isothiocyanate (FITC; Invitrogen) before use.

Cell culture: Fresh, unprocessed human bone marrow $(25 \mathrm{~mL})$ was purchased from Cambrex. Mononuclear cells were isolated by utilizing a density gradient centrifugation in Ficol-Paque solution (GE Healthcare) at $400 \times g$ for 30 min. Mononuclear cells derived from human umbilical cord blood cells were gifted from Prof. Larry Lasky's lab (Ohio State University). For CD34+/CD4- HSC culture, we first depleted CD4+ cells from mononuclear cells and then separated $\mathrm{CD} 34+$ cells utilizing magnetic-activated cell sorting kits (Milteney Biotech). Approximately 1-1.5 × 10 ${ }^{6}$ CD34+/CD4HSCs were collected. Isolated mononuclear cells and HSCs were cultured in serum-free medium (StemSpan; Stemcell Technologies) supplemented with $20 \mathrm{ng} \mathrm{mL}^{-1}$ of Flt-3 ligand, stem cell factor, and thrombopoietic (Stemcell Technologies). Five scaffolds were put in a $10 \mathrm{~mL}$ rotary cell culture vessel (Synthecon) and approximately $0.2 \times 10^{6} \mathrm{HSCs}$ were seeded in the vessel. The rotary culture was maintained at $15 \mathrm{rpm}$ for 4 weeks and culture medium was changed every 3 days. The experiment was repeated three times and similar results were obtained.

Characterization: Cell morphology and distribution across ICC scaffolds were characterized under SEM. HSC ICC scaffolds were 
fixed with $2.5 \%$ glutaraldehyde solution overnight and dehydrated through a series of ethanol solution concentrations of $50 \%, 70 \%$, $90 \%, 95 \%$, and $100 \%$ for $10 \mathrm{~min}$. Dehydrated samples were further freeze-dried (Labcono) and then coated with gold using a sputter coater (Venton Vaccum). FEI Nova Nanolab (University of Michigan Electron Microbeam Analysis Laboratory) was used for SEM imaging.

The HSC differentiation stage was determined by surface marker analysis. HSC-growing ICC scaffolds were fixed with $4 \%$ formaldehyde and blocked with $10 \%$ normal goat serum to prevent non-specific binding of antibodies. Primary antibodies CD34, CD117, CD4, CD7, CD8, and TCR $\alpha$ (Santa Cruz biotechnology) diluted in blocking solution were applied for $1 \mathrm{~h}$. After washing, diluted secondary antibodies conjugated to Alexa Fluor 488 and 568 dyes (Invitrogen) were introduced for $1 \mathrm{~h}$. The antibody-stained scaffolds were visualized under confocal microscopy (Leica SP2) with $10 \times$ and $20 \times$ objectives.

\section{Keywords:}

layer-by-layer assembly · ligands · scaffolds · stem cells · thymus

[1] A. C. Janeway, P. Travers, M. Walport, J. M. Schlomchik, Immunobiology: The immune system in health and disease, Garland Science Publishing, New York 2004.

[2] W. van Ewijk, B. Wang, G. Hollander, H. Kawamoto, E. Spanopoulou, M. Itoi, T. Amagai, Y.-F. Jiang, W. T. V. Germeraad, W.-F. Chen, Y. Katsura, Semin. Immunol. 1999, 11, 57.

[3] A. Bhandoola, H. von Boehmer, H. T. Petrie, J. C. Zúñiga-Pflücker, Immunity 2007, 26, 678.

[4] H. T. Petrie, Nat. Rev. Immunol. 2003, 3, 859.

[5] M. Ciofani, J. C. Zuniga-Pflucker, Annu. Rev. Cell Dev. Biol. 2007, 23, 463.

[6] H. T. Petrie, J. C. Zuniga-Pflucker, Annu. Rev. Immunol. 2007, 25, 649.

[7] E. J. Jenkinson, G. Anderson, J. J. Owen, J. Exp. Med. 1992, 176, 845.

[8] E. Robey, Annu. Rev. Immunol. 1999, 17, 283.

[9] A. W. Duncan, F. M. Rattis, L. N. DiMascio, K. L. Congdon, G. Pazianos, C. Zhao, K. Yoon, J. M. Cook, K. Willert, N. Gaiano, T. Reya, Nat. Immunol. 2005, 6, 314.

[10] J. C. Zuniga-Pflucker, Nat. Rev. Immunol. 2004, 4, 67.

[11] T. M. Schmitt, J. C. Zúñiga-Pflücker, Immunity 2002, 17, 749.

[12] R. N. La Motte-Mohs, E. Herer, J. C. Zuniga-Pflucker, Blood 2005, 105, 1431.

[13] N. Panoskaltsis, A. Mantalaris, J. H. D. Wu, J. Biosci. Bioeng. 2005, 100, 28.

[14] J. E. Nichols, J. Cortiella, J. Lee, J. A. Niles, M. Cuddihy, S. Wang, J. Bielitzki, A. Cantu, R. Mlcak, E. Valdivia, R. Yancy, M. L. McClure, N. A. Kotov, Biomaterials 2009, 30, 1071.
[15] D. Marshall, J. Bagley, P. Le, K. Hogquist, S. Cyr, E. von Schild, M. Pykett, M. Rosenzweig, J. Hematother. Stem Cell Res. 2003, 12, 565.

[16] M. C. Poznansky, R. H. Evans, R. B. Foxall, I. T. Olszak, A. H. Piascik, K. E. Hartman, C. Brander, T. H. Meyer, M. J. Pykett, K. T. Chabner, S. A. Kalams, M. Rosenzweig, D. T. Scadden, Nat. Biotechnol. 2000, $18,729$.

[17] J. Lee, M. J. Cuddihy, N. A. Kotov, Tissue Eng, Part B 2008, 14, 61.

[18] B. Varnum-Finney, L. Xu, C. Brashem-Stein, C. Nourigat, D. Flowers, S. Bakkour, W. S. Pear, I. D. Bernstein, Nat. Med. 2000, 6, 1278.

[19] B. Varnum-Finney, L. Wu, M. Yu, C. Brashem-Stein, S. Staats, D. Flowers, J. D. Griffin, I. D. Bernstein, J. Cell Sci. 2000, 113, 4313.

[20] B. Varnum-Finney, C. Brashem-Stein, I. D. Bernstein, Blood 2003, $101,1784$.

[21] S. Taqvi, L. Dixit, K. Roy, J. Biomed. Mater. Res, Part A 2006, 79A, 689.

[22] M. H. Dallas, B. Varnum-Finney, P. J. Martin, I. D. Bernstein, Blood 2007, 109, 3579.

[23] M. H. Dallas, B. Varnum-Finney, C. Delaney, K. Kato, I. D. Bernstein, J Exp. Med. 2005, 201, 1361.

[24] N. A. Kotov, Y. Liu, S. Wang, C. Cumming, M. Eghtedari, G. Vargas, M. Motamedi, J. Nichols, J. Cortiella, Langmuir 2004, 20, 7887.

[25] J. Lee, S. Shanbhag, N. A. Kotov, J. Mater. Chem. 2006, 16, 3558.

[26] G. Decher, Science 1997, 277, 1232.

[27] S. Shanbhag, J. Lee, N. Kotov, Biomaterials 2005, 26, 5581.

[28] S. Shanbhag, S. Wang, A. N. Kotov, Small 2005, 1, 1208.

[29] Z. Tang, Y. Wang, P. Podisadlo, N. A. Kotov, Adv. Mater. 2006, 18, 3203.

[30] D. S. Lidke, P. Nagy, R. Heintzmann, D. J. Arndt-Jovin, J. N. Post, H. E. Grecco, E. A. Jares-Erijman, T. M. Jovin, Nat. Biotechnol. 2004, 22, 198.

[31] V. H. Fan, A. Au, K. Tamama, R. Littrell, L. B. Richardson, J. W. Wright, A. Wells, L. G. Griffith, Stem Cells 2007, 25, 1241.

[32] W. Han, Q. Ye, M. A. S. Moore, Blood 2000, 95, 1616.

[33] M. Onda, K. Ariga, T. Kunitake, J. Biosci. Bioeng. 1999, 87, 69.

[34] A. Nadiri, S. Kuchler-Bopp, H. Mjahed, B. Hu, Y. Haikel, P. Schaaf, J.-C. Voegel, N. Benkirane-Jessel, Small 2007, 3, 1577.

[35] F. Caruso, K. Niikura, D. N. Furlong, Y. Okahata, Langmuir 1997, 13, 3427.

[36] Z. Tang, N. A. Kotov, S. Magonov, B. Ozturk, Nat. Mater. 2003, 2, 413.

[37] C. N. Cavasotto, R. A. Abagyan, J. Mol. Biol. 2004, 337, 209.

[38] M. Brenner, Nat. Med. 2000, 6, 1210.

[39] J. Kimble, P. Simpson, Annu. Rev. Cell Dev. Biol. 1997, 13, 333.

[40] K. C. Wood, H. F. Chuang, R. D. Batten, D. M. Lynn, P. T. Hammond, Proc. Natl. Acad. Sci. U. S. A. 2006, 103, 10207.

[41] P. Podsiadlo, M. Michel, J. Lee, E. Verploegen, N. Wong Shi Kam, V. Ball, J. Lee, Y. Qi, A. J. Hart, P. T. Hammond, N. A. Kotov, Nano Lett. 2008, 8, 1762.

[42] S. Srivastava, V. Ball, P. Podsiadlo, J. Lee, P. Ho, N. A. Kotov, J. Am. Chem. Soc. 2008, 130, 3748.

Received: August 9, 2008 Revised: February 17, 2009 Published online: March 30, 2009 